Review Article

\title{
Extracellular Vesicles with Possible Roles in Gut Intestinal Tract Homeostasis and IBD
}

\author{
Xin Chang, ${ }^{1,2}$ Shu-Ling Wang, ${ }^{1,2}$ Sheng-Bing Zhao, ${ }^{1,2}$ Yi-Hai Shi, ${ }^{1}$ Peng Pan, ${ }^{1,2}$ Lun Gu, ${ }^{1,2}$ \\ Jun Yao $\left({ }^{3},{ }^{3}\right.$ Zhao-Shen Li ${ }^{1},{ }^{1,2}$ and Yu Bai ${ }^{1,2}$ \\ ${ }^{1}$ Department of Gastroenterology, Gongli Hospital, The Second Military Medical University, Shanghai, China \\ ${ }^{2}$ Department of Gastroenterology, Changhai Hospital, Second Military Medical University/Naval Medical University, \\ Shanghai, China \\ ${ }^{3}$ Department of Gastroenterology, The Second Clinical Medical College, Jinan University, Shenzhen, China
}

Correspondence should be addressed to Jun Yao; yj_1108@126.com, Zhao-Shen Li; li.zhaoshen@hotmail.com, and Yu Bai; baiyu1998@hotmail.com

Received 18 September 2019; Revised 17 December 2019; Accepted 24 December 2019; Published 13 January 2020

Academic Editor: Soh Yamazaki

Copyright (C) 2020 Xin Chang et al. This is an open access article distributed under the Creative Commons Attribution License, which permits unrestricted use, distribution, and reproduction in any medium, provided the original work is properly cited.

\begin{abstract}
The intestinal tract consists of various types of cells, such as epithelial cells, Paneth cells, macrophages, and lymphocytes, which constitute the intestinal immune system and play a significant role in maintaining intestinal homeostasis by producing antimicrobial materials and controlling the host-commensal balance. Various studies have found that the dysfunction of intestinal homeostasis contributes to the pathogenesis of inflammatory bowel disease (IBD). As a novel mediator, extracellular vesicles (EVs) have been recognized as effective communicators, not only between cells but also between cells and the organism. In recent years, EVs have been regarded as vital characters for dysregulated homeostasis and IBD in either the etiology or the pathology of intestinal inflammation. Here, we review recent studies on EVs associated with intestinal homeostasis and IBD and discuss their source, cargo, and origin, as well as their therapeutic effects on IBD, which mainly include artificial nanoparticles and EVs derived from microorganisms.
\end{abstract}

\section{Introduction}

The homeostasis of the intestinal tract is the most complex homeostasis within the human body due to the direct exposure to the digestive residue, millions of pathogens, and high concentrations of foreign antigens [1]. During this process, the intestinal mucosal barrier plays a pivotal role in detecting and clearing the pathogenic microbial debris, while maintaining a peaceful coexistence with them. As for the intestinal defense system, it mainly consists of three parts, including the mucus layer, intestinal epithelial cells (IECs), and other immune cells, such as lymphocytes and macrophages that are associated with the innate immune system. Additionally, effective communication among these cells plays a critical role in maintaining the intestinal homeostasis, which is mainly mediated by extracellular factors and receptors, such as growth factor and its receptor tyrosine kinase $[2,3]$. How- ever, in recent decades, extracellular vesicles (EVs) have been recognized as a novel mediator not only for the cell-to-cell but also for the organism-to-cell interaction [4-6]. In addition, the mammalian intestine encounters about 10 trillion $\left(10^{13}\right)$ microbes which is approximately equal to ten times the number of our total cells, and the whole genome from this microorganism even exceeds that of the entire human genome by 150- to 400-fold [7]. As a result, the coexistence with gut microbiota plays a significant role in maintaining intestinal homeostasis, which has been recognized as a major determinant to our health $[8,9]$.

Microbiota-derived EVs carry a large diversity of compounds that can affect various pathways in the host. Emerging evidence has demonstrated the role of EVs in bacterial survival and host interaction [6]. EVs are submicroncirculating vesicles found in all bodily fluids and in all species, including bacteria. Eukaryotic cells' EVs originate from 
the process of plasma membrane budding or fusion of multivesicular endosomes with the plasma membrane. Relatively, EVs derived from Gram-positive and Gram-negative bacteria may disperse in extracellular space by outward budding of the prokaryotic membrane [10-12]. In past reviews, the EVs tend to be divided into three main subsets known as exosomes, microvesicles (MVs), and apoptotic bodies [13, 14]. Their intrinsic heterogeneity can separate and characterize them with varying sizes, molecular patterns, and triggering mechanisms. Exosomes $(40-150 \mathrm{~nm})$ are produced via a lyso-endosomal system. MVs (100-1000 nm) are generated through the direct budding of the cell plasma membrane in a calcium-dependent process. Apoptotic bodies $(>2000 \mathrm{~nm})$ are released during cell apoptosis and are the most heterogeneous type, with a diverse morphology. However, this classification neglects bacteria-released membrane vesicles (20-400 nm) which are regarded as MVs or outer membrane vesicles (OMVs) based on whether they are Gram-negative or Gram-positive $[15,16]$. In this review, bacteria-released membrane vesicles were classified as EVs due to the mechanism for any organisms' intercellular communication. Therefore, EVs are evolutionarily conserved across eukaryotes, bacteria, and archaea. Here, we highlight specific paradigms of cell-to-cell and organism-to-cell communication in intestinal homeostasis. Additionally, we provide a brief update on the clinical application of EVs as delivery vehicles as well as the sources of diagnostic markers.

1.1. EVs. EVs are found in most physiological fluids, including urine, breast milk, and bile [17]. Additionally, EVs can also be collected from cell culture supernatant. EVs within the intestinal tract can be derived from cells, organisms, or physiological fluids, such as succus entericus. A previous study found that patients with malignant common bile stenoses contained significantly higher concentrations of EVs than healthy controls in bile samples, indicating that characteristics of EVs vary at different states of the body [18]. Furthermore, EVs contain bioactive cargo, such as nucleic acids (DNA, mRNA, microRNA, and other noncoding RNAs), proteins (receptors, transcription factors, enzymes, and extracellular matrix proteins), and lipids which can regulate the functions of the recipient cell [19-21].

1.2. Classification of EVs. Based on present studies regarding the biogenesis and size of EVs, three categories of EVs as well as several terms, including microvesicles, exosomes, ectosomes, oncosomes, and outer membrane vesicles, are presented [22]. Exosomes refer to EVs ranging between 40 and $150 \mathrm{~nm}$ in diameter and are produced from the multivesicular endosome pathway. While those in the range of 100 to $1000 \mathrm{~nm}$ are microvesicles or microparticles derived from plasma membrane. Microvesicles that separated at approximately 10 to $14,000 \mathrm{~g}$ are heterogeneous. In contrast, microvesicles separated at 100,000 g are homogeneous [17, 23-25]. Apoptotic bodies with large populations originate from membrane blebbing and cellular disassembly from cell fragmentation when the cytoskeleton breaks at the beginning of apoptosis. Recently, larger-size EV subpopulations (1-10 $\mu \mathrm{m}$ diameter) were distinguished from highly migra- tory cancer cells and were termed as oncosomes due to their distinguishing biomolecules and unique extraction methods (Figure 1). As mentioned above, the present classification is based on the eukaryote system excluding the bacteriareleased membrane vesicles. However, the shedding of membrane vesicles is ubiquitous in bacteria. The production of OMVs was first discovered in Gram-negative bacteria in 1963 [26]. They were identified as OMVs due to them originating from the controlled blebbing of the outer membrane of Gram-negative bacteria. Moreover, recent work has shown the vesicles and MVs of bacteria refer to those of archaea and Gram-positive bacterial origin [6, 27]. OMVs refer to those originating from Gram-negative bacteria with a diameter of about $20-400 \mathrm{~nm}$, while MVs are cytoplasmic membranes of Gram-positive bacteria with a diameter typically of 20-150 nm [28]. Both of OMVs and MVs can carry DNA, sRNA, proteins, and other factors to the recipient cells [29].

1.3. Biogenesis and Characteristics of EVs. Exosomes originate through the lyso-endosome pathway. Exosomes are released upon the fusion of multivesicular bodies (MVBs) with the plasma membrane. MVBs are vesicular entities generated in the maturation process of the early endosomes formed by plasma membrane invagination. Within the cytoplasm, the membrane of MVBs forms intraluminal vesicles (ILVs) by inward budding. After MVBs fuse with the plasma membrane, they release inside ILVs, which are called exosomes [19, 30, 31]. According to the International Society for Extracellular Vesicles (ISEV), there are minimal requirements to claim the presence of exosome isolation; several experiments need to be conducted to characterize the existence of the exosomes, such as electron microscopy, concentrationmonitoring techniques, and western blotting [14]. The cupshaped lipoidal vesicle structure is the typical feature of EVs under the electron microscope. In biochemistry, the tetraspanin superfamily was previously thought to be a specific marker of exosomes. However, MV has also been reported to bear CD63, CD9, and CD81 tetraspanin proteins in recent years. In multiple studies, investigations concerning Alix and TSG101 involving the exosome forming process and heat shock proteins HSC70 and HSP90 have also been carried out with exosomes [32].

According to previous studies, microvesicles are mainly derived from plasma via a calcium-regulated pathway which requires lipid formation for budding out [33]. As mentioned above, apart from exosomes, MVs also contain the tetraspanin protein family (CD9, CD63, and CD81), thus indicating the significance of these proteins in the budding and fusion of the membrane [34]. Moreover, MVs can also generate a more heterogeneous subpopulation of extracellular vesicles carrying surface markers and receptors from their parental cell, which takes part in intercellular communication and capacitates their identification in the laboratory [35]. The studies included in this review do not discriminate endosomederived from plasma membrane-derived EVs. In this review, we use the term "EVs" rather than the term in the cited literature, thereby no longer distinguishing an endosomal or plasma membrane origin. 


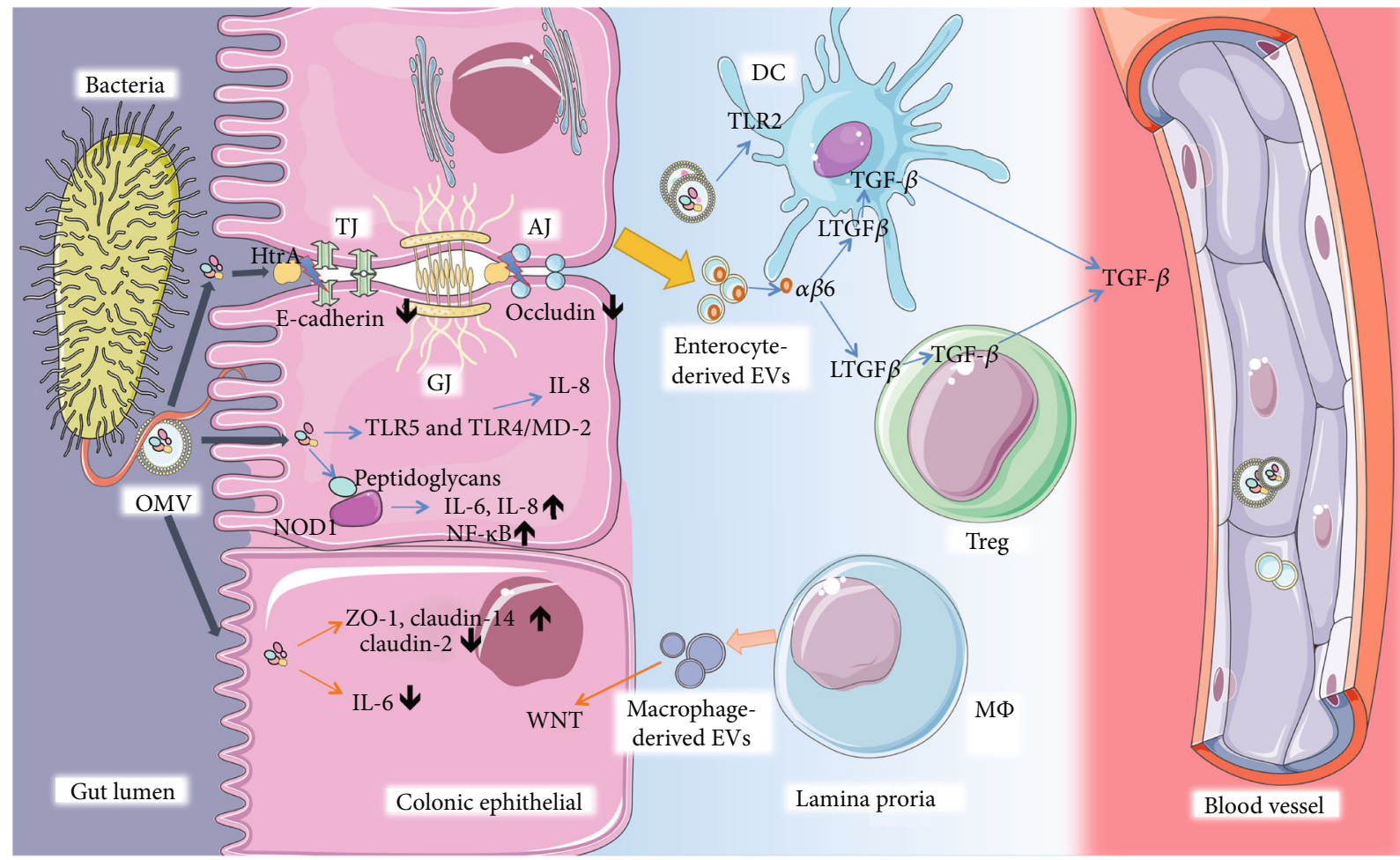

FIGURE 1: Classification of extracellular vesicles according to the mechanism of generation. Extracellular vesicles include exosomes, microvesicles, apoptotic bodies, out membrane vesicles, and membrane vesicles (not shown in the figure) in this review. Exosomes are produced by budding from multivesicular bodies. Microvesicles are generated intracellularly from the extracellular membrane. Apoptotic bodies are originated upon cell fragmentation during apoptotic cell death.

As a result, these tetraspanin proteins, including CD9, CD63, and CD81, were regarded as markers to evaluate the purity of the molecules after isolation. Additionally, physical properties, such as particle size, were also characteristic during the process of isolation via ultracentrifugation, density gradient separation, and polymer-based precipitation methods.

\section{EVs in IBD}

2.1. Microbiota-Derived EVs. EVs are produced by all domains of life, including microorganism Gram-negative and Gram-positive bacteria, archaea, fungi, and protozoa $[28,36]$. The alterations in microbiota colonizing intestines have been implicated in the pathogenesis and development of many diseases and particularly in IBD $[37,38]$. The balance between host and commensal microbe in the intestine is the key to maintaining a healthy human state, as they can regulate the maturation and functions of IECs and various immune cells. EVs released from both pathogenic and commensal bacteria are important regulators of host-pathogen communication that regulate immunomodulation and the corresponding signaling pathways. For instance, Pseudomonas aeruginosa OMV-mediated short RNAs (sRNAs) reduced the secretion of IL-8 in IECs which were induced by lipopolysaccharide (LPS). The enriched sRNA52320 can attenuate OMV-induced KC cytokine secretion and neutrophil infiltration [39]. On the contrary, the EVs derived from the physiological fluids may also influence the intestinal microbiota. A previous study used EVs from the sera of Toll-like receptor (TLR) 2 knockout mice and wild-type mice to interact with Lactobacillus or Bifidobacterium which are common bacteria in the gut. The study found that EVs significantly reduced the activity of TLR2/6 both in Bifidobacterium and Lactobacillus, thus contributing to the aggregation of pathogens [40]. EVs were first discovered over 40 years ago. In 1967, Chatterjee and Das revealed the excretion of cell wall material in Vibrio cholera by electron microscopy. They found that Neisseria meningitides released endotoxins in the form of cell wall blebs in vivo [41]. EVs produced by commensal bacteria in the gastrointestinal tract of animals are distributed throughout the gut lumen with a variety of biomolecules, nucleic acids, enzymes, toxins, and metabolites. The engagement of extracellular products from commensal bacteria in immunomodulatory activities has been noted since 1967 [41]. However, the mechanism involved has not yet been studied completely or systematically.

Sometimes, microbiota-derived EVs serve as bad factors in digestive tract homeostasis. Helicobacter pylori $(\mathrm{Hp})$ infection can lead to gastritis, ulceration, or malignancy due to a degree of adhesion to the epithelium. Furthermore, in 2003, Ismail et al. revealed that there is no need for $\mathrm{Hp}$ to directly contact the epithelium cell to cause gastritis and that OMVs from $\mathrm{Hp}$ could be accepted by the host cells and further stimulate various responses independently [42]. Recently, EVs from enterohemorrhagic Escherichia coli (EHEC) O157 during growth were found to stimulate the production of interleukin-8 (IL-8) in IECs via the TLR5 and TLR4/MD-2 complex signaling pathway [43]. They also deliver the 
hemolysin from EHEC to microvascular endothelial cells and mitochondria, thus triggering apoptosis [44]. OMVs from bacteria are the cargo of many various ligands of pattern recognition receptors (PRR), including DNA, RNA, lipoproteins, LPS, and peptidoglycan, which initiate proinflammatory signaling cascades. OMVs from commensal Escherichia coli containing peptidoglycans that can colonialize with Nucleotide Binding Oligomerization Domain Containing 1 (NOD1), trigger the NOD1 signaling pathway, and improve the expression of NF- $\kappa \mathrm{B}$, IL- 6 , and IL- 8 [45]. In addition, OMVs can enter the IECs via clathrindependent endocytosis and give rise to DNA damage [46]. In dextran sulfate sodium- (DSS-) induced colitis, the gut microbiota regulate intestinal UDP-glucuronosyltransferase 1A1 (UGT1A1) through secreting cargo that can interact with epithelial cells directly [47]. Vibrio cholera secrets EVassociated Zn-dependent hemagglutinin protease (HAP), and cholera toxins are transported to human IECs to induce dose-dependent apoptosis [48, 49]. Furthermore, these EVs internalized by IECs induce the expression of IL-8, GM-CSF, and chemokines, such as CCL2, CCL20, and thymic stromal lymphopoietin, in epithelial cells by activating the MAPK and NF- $\kappa$ B pathways in a NOD1-dependent manner [50].

In addition, microbiota-derived EVs may help to maintain the homeostasis of the intestinal tract. It is generally known that the integrity of the gastrointestinal epithelial layer, consisting of the physical and biochemical barrier, is critical in fighting against various toxins and pathogens. Apart from these cells, intestinal microbes, especially probiotic bacteria, can modulate barrier integrity by reducing gut epithelial proinflammation, reinforcing tight junctions, and other reciprocal interactions among commensal bacteria, the epithelium, and the mucosal immune system. Escherichia coli $\mathrm{C} 25$, the first colonized bacteria in the intestine, elicit a mild proinflammatory effect on host epithelial cells with upregulated TLR in vitro, which is considered to be the mediator of a rapid but more controllable reaction to pathogenic bacteria in vivo [51]. Probiotic Escherichia coli Nissle $1917(\mathrm{EcN})$ act as beneficial colonizers in the human gut by secreting the protein $\mathrm{TcpC}$ to regulate the expression of tight junction protein in IBD [52]. However, the independence of TcpC has been verified in probiotic E. coliderived EVs. In 2016, Alvarez et al. illustrated that EVs from both EcN and ECOR63 have a strengthening ability based on TcpC. EVs isolated from these probiotics can promote the upregulation of ZO-1 and claudin-14 and downregulation of claudin-2, thus helping the reinforcement of the epithelial barrier, while the specific mechanism has not yet been illustrated [53]. EVs from Bacteroides thetaiotaomicron (BtMinpp) may protect enzymes from degradation by gastrointestinal proteases and promote intracellular $\mathrm{Ca}^{(2+)}$ signaling, thus maintaining the physiological responses of the digestive system [54]. Meanwhile, EVs isolated from intestinal microbiota have been evaluated in an experimental IBD model. Owing to the complexity of the gut microbiota, their roles are different: EVs from E. coli induce colon epithelial cells to release the proinflammatory cytokine IL6, while Akkermansia muciniphila can alleviate this. The oral application of EVs from A. muciniphila ameliorates the levels of inflammation both in LPS-stimulated macrophages and IECs [55].

However, pathogenic EVs can disrupt intestinal barrier integrity and exaggerate the invasion of harmful components into the submucosa, thus contributing to the pathogenesis of IBD. Campylobacter jejuni has been detected in many tissues, such as lamina propria, and blood. Recently, C. jejuni was reported to cleave cell-to-cell junction factors, such as E-cadherin, and occlude facilitating the invasion of pathogens into IECs via serine protease HtrA and bacterial EVs $[56,57]$. The toxicity of HtrA proteins and their orthologues are nonnegligible in both prokaryotes and eukaryotes [58]. The function of E-cadherin to establish and maintain epithelial integrity has been discussed in many studies [59, 60]. Deleting the HtrA protein in C. jejuni can alter Ecadherin shedding [61]. Furthermore, pretreatment with methyl-beta-cyclodextrin partially blocks OMV-induced host immune responses, demonstrating the effect of lipid rafts on host cell plasma membranes during interactions with $C$. jejuni OMVs [62].

2.2. Enterocyte-Derived EVs. The essential function of the intestinal epithelium is to form a barrier regulating the interactions with luminal contents. It can also act as the underlying immune system, regulating the inflammation response. Through complex communication with the pathogens and the immune system, IECs maintain intestinal homeostasis.

\subsection{EVs Derived from IEC Regulation of Gut Immune} Cells. IECs promote the development of dendritic cells (DCs) and macrophages with tolerogenic properties by producing numerous immunoregulatory signals, including TGF- $\beta$, thymic stromal lymphopoietin (TSLP), and retinoic acid [63-65]. Professional antigen-presenting cells (APC) have been verified to secrete major histocompatibility complex- (MHC-) bearing vesicles called exosomes, which are a subset of EVs [66]. Although IECs are not primarily APCs, they constitutively express MHC I, MHC II, and HLA-DM localized in vesicular structures from biopsies and HT-29 cells [67]. Additionally, EVs from these enterocyte cells can be released from either the apical or basolateral side. They preferentially interact with DCs and potentiate antigenpresenting capacity [68]. The fact that IECs release EVs has been known for more than ten years, and this investigation complemented the lack of direct contact between IEC and CD4+ T-cells [69]. Their EVs express immunomodulatory molecules, such as major histocompatibility complex (MHC) class I and class II molecules, whose expression levels are much higher in inflammatory conditions compared with basal conditions [68-70]. MHC II is essential in initiating adaptive immunity; its upregulation during B-cell development suggests its role in consolidating B-cell maturation [71]. The adaptive immune response is related to the high expression of MHC I molecules in esophageal adenocarcinoma development [72]. Except for the normal antigenpresenting molecules enriched on EV surfaces, EVs derived from IECs specifically display A33 antigens used to identify the origin of the EVs $[67,68,73]$. EVs derived from IECs have been demonstrated to be necessary for tolerogenic 
immune cells and directing appropriate innate and adaptive immune cell responses in both physiology and pathological states. Additionally, tolerogenic DCs are considered indispensable for maintaining intestinal homeostasis [1]. EVs derived from IECs carrying $\alpha \beta 6$ activate LTGF $\beta$ in intestinal tolerogenic DCs and Tregs, which first produces TGF- $\beta$. After internalizing the EVs, DCs improve the expression of TGF- $\beta$ and finally induce the Treg cell and drive tolerogenic responses [74]. Epithelial EVs may participate in this tolerogenic process directly. In 2001, Karlsson et al. named exosome-like structures as tolerosomes, which were isolated from rat IECs and can induce antigen-specific tolerance when administered to naive recipient rats intraperitoneally [75]. In 2016, Jiang et al. demonstrated that EVs originate from IECs containing TGF- $\beta$ inhabited CD $4+$ cell proliferation under physiological conditions [76]. In posttrauma immune dysfunction, the expression of CD63 (a specific marker of exosome) and the epithelial cell-specific marker epithelial cell adhesion molecule (EpCAM) were improved greatly, illustrating that EVs from IECs induce DC apoptosis, suppress DC maturation, and inhibit the Ag-presenting function of DCs [77]. The EpCAM induces the homophilic interaction molecule between IECs and intraepithelial lymphocytes in the physical mucosal epithelium and regulates the positive effect of EVs on the intestinal tract immune balance $[76,78]$.

\subsection{IEC-Derived EVs Promoting Repairment and Regulating} the Inflammatory Response. EVs originated from IECs carry the component promoting epithelial healing, coinciding with the resolution of inflammation. Annexin A1 (ANXA1) facilitates the repair of intestinal mucosal wounds in a murine model of colitis, and their release is elevated during wound closure [79, 80]. In 2015, Giovanna et al. reported that EVs derived from IECs containing ANAXA1 can be used to activate wound repair circuits and promote epithelial restitution. During mucosal repair, ANAXA1 in EVs acted as an endogenous mediator of wound healing by binding to formyl peptide receptors (FPRs) expressed on responsive cells [81]. In 2018, Zhang et al. identified that EVs isolated from the mucosal-luminal interface of IBD patients contained defense protein MPO [82]. The MPO function is to induce the oxidation reaction by producing reactive oxidants, such as hypohalous acids [83-85]. The increased level of oxidative stress can withstand the microbes in the gut of patients with IBD [86]. However, EVs isolated from the intestinal lumen fluid of patients with IBD had a proinflammatory effect on IECs in vitro [87]. This discrepancy may be caused by the source of the EVs. This is because intestinal lumen fluid is quite different from the aspirate of the mucosal-luminal interface. The alteration of enterobacteria has already been linked with gut-associated inflammation, which is itself a crucial risk factor for colon cancer. In 2015, Deng et al. revealed that enterotoxigenic Bacteroides fragilis secreted EVs that could induce the production of intestinal mucosa-derived EVs containing elevated levels of sphingosine-1-phosphate, CCL20, and prostaglandin E2 [88]. Additionally, CCL20 and prostaglandin E2 recruit Th17 cells through the MyD88-mediated pathway [88]. Several studies have demonstrated the role of sphingosine-1-phosphate in tumorigenesis [89-91]. These studies also implicated a possible role of EVs derived from normal intestinal mucosa in suppression of CCL20 and other proinflammatory cytokines [88].

2.5. Immune Cell-Derived EVs. Previous studies have proven the link between the abnormal immune responses and IBD. Both the innate and adaptive immune responses contribute greatly to the IBD pathogenesis. The innate immune responses act faster to trigger the phagocytic responses and antigen presentation, along with initiating the adaptive immune system. These involve various immune cells, such as the macrophages, DCs, neutrophils, and monocytes. Several studies have shown the immune-stimulatory effects of the EVs from DCs [92]. The inhibition of T-cell proliferation by EVs derived from DCs has been proposed to play a key role in suppression of the inflammation-related disease, such as IBD $[93,94]$. As compared to the nongene-modified BMDC, TGF- $\beta 1$ gene-modified BMDC can lead to the release of immunosuppressive EVs that contain high levels of TGF- $\beta 1$ and elicit stronger inhibitory effects on the Tcell proliferation [95]. In addition, much work has demonstrated EVs from conditioned DC might promote IBD in remission. The EVs derived from DCs treated with S. japonicum-soluble egg antigens or IL-10 play a protective role during acute IBD development $[94,96,97]$. Furthermore, EVs from other immune cell can influence disease progression in different ways. Intestinal mucosa polymorphonuclear neutrophil (PMN) infiltration is common in IBD. During the infiltration of these immune cells, myeloperoxidase (MPO) can be released into the extracellular environment. The MPO release is common in acute and chronic inflammation. During the progression of IBD, MPO can damage the gut barrier. In 2019, Thomas et al. explored a new regulation mechanism between MPO and PMNs during inflammation [98]. With the help of EVs, MPO can be protected and delivered to IECs. The tissue-infiltrating PMNs together with MPO enhanced the inflammatory response and inhibited the wound closure through the regulation of the IEC migration and proliferation [98]. Similarly, Butin-Israeli et al. confirmed the role of EVs armed with the proinflammatory microRNAs in mediating the accumulation of the doublestrand breaks (DSBs) in degenerated colonic epithelium [99]. miR-23a and miR-155 in EVs can induce lamin B1dependent replication fork collapse and inhibit homologous recombination (HR) by targeting the HR-regulator RAD51 [99]. The role of PMN-derived EVs in promoting DSB formation and suppressing DSB repair through the downregulation of lamin B1 and Rad51 was confirmed again in 2019 [100]. Furthermore, there is another explanation for PMN transepithelial migration. Butin-Israeli et al. showed that during transepithelial migration, the EVs derived from PMN were deposited on the IECs, leading to the loss of epithelial cadherins while enhancing the PMN recruitment [101]. Meanwhile, the other immune cell-derived EVs exhibited high immunomodulatory capacity to be attractive agents. EVs released by the granulocytic myeloid-derived suppressor cells caused a decrease in the proportion of Th1 cells and an increase in the proportion of regulatory $\mathrm{T}$-cells 
in colitis mice [102]. WNT/ $\beta$-catenin signaling, one of the major sources of WNT ligands [103, 104], is significant for intestinal homoeostasis and the intestinal epithelium. Macrophage-derived EVs can rescue the intestinal stem cells and enhance the survival rate of the enterocytes after radiation injury through the regulation of WNT function [105].

\section{The Clinical Potential of EVs in IBD}

As previously discussed, scientific interest in EVs has been stimulated due to their key roles in cell-cell and cellorganism communication. There is an urgent need to convert these fundamental achievements into clinical applications. Therefore, an increasing number of studies regarding EVs have been proposed to explore its role as a source of diagnostic and prognostic markers or as promising pharmaceutical vehicles.

3.1. Clinical Potential of EVs as Biomarkers. In recent years, multiple studies have investigated more precise markers of cancer. One essential approach aimed at diagnosing the development of cancer is based on the cargo of EVs. In 2014, Li et al. confirmed that using EVs was more amenable to the development of a disease marker panel rather than whole bile [106]. Likewise, a large body of work focusing on purifying EVs, increasing the abundance of cargo, and decreasing heterogeneity of the sample has been produced [107-109]. In 2018, a laboratory-built high-sensitivity flow cytometer was established for quantitative multiparameter analysis of single EVs. According to the corresponding report, the challenge of profiling and sizing the individual EVs was conquered through this new method. The author used this method to analyze blood samples from patients with colorectal cancer and healthy controls, and they obtained an accurate resolution and profile of EVs, thereby identifying CD147-positive EVs as a sensitive biomarker for colorectal cancer [110]. Similarly, miRNA in EVs can be an essential biomarker for the detection of disease recurrence. A previous study showed that the miR-17-92 cluster is highly expressed in microRNAs in patients with a poor prognosis [111]. IBD is known to potentially increase the risk of developing cancer [112-114]. However, there is a lack of promising biomarkers for the complicated surveillance of IBD. In 2015, Polytarchou et al. demonstrated that miR214 is associated with the progression of IBD, and reducing its expression can slow the development of colitis and colitis-associated cancer in mice [115]. Interestingly, miR214 also has been detected in the EVs of many gastroenterology cancers $[116,117]$. In the meantime, isolating miRNAs from exosomes has been proven to be more stable and reliable than biomarkers in many studies [118-120]. These findings imply the function of EVs to monitor the cancer progression of IBD.

Circulating pathogenesis-related EVs have emerged as promising biomarkers to monitor disease development and as novel targets for future anti-inflammation therapies in IBD. In 2017, Zheng et al. investigated the high sensibility of salivary exosomal PSMA7 on IBD diagnosis [121]. This study identified the proteins within EVs by using a liquid chromatograph-mass spectrometer, and PSMA7 was shown to be associated with inflammation and immune response as well as depressive disorder in many studies $[122,123]$. Rab proteins of the GTPase family are involved in selective packaging and docking at the plasma membranes of EVs [124]. With regard to the intestinal immune balance, the numbers of RAB27A- and RAB27B-positive immune cells increased in the colonic mucosa of patients with active ulcerative colitis (UC) compared to the healthy controls [125, 126]. Double knockdown of Rab27A and Rab27B led to interference in protecting mice from T-cell-transfer-induced colitis, which authenticated the crucial role of Rab27mediated EVs in the treatment of IBD [127, 128]. All these findings indicate that EV biogenesis acts as a key strategy for the diagnosis and/or therapeutic potential of EVs in IBD.

3.2. The Clinical Potential of EVs on Treatment. Targeting specific cargo and transmembrane integrin of EVs might alleviate the inflammation of intestines. In the intestinal tract, the interaction between IEC and EVs is weaker in EpCAMknockout mice. In the meantime, the protective effect of EVs has been decreased in IBD [76]. Genetic material within EVs shows its potential therapeutic role in IBD. Bone marrow mesenchymal stem cells (BMSCs) transfected with lentivirus to overexpress miR-200b can release EVs packaged with miRNA-200b. The miR-200b-EVs significantly suppressed ZEB1 and ZEB2 to reverse the morphology in TGF- $\beta 1$-treated IEC- 6 cells and ameliorate the TNBS(2,4,6-trinitrobenzene sulfonic acid-) induced colon fibrosis histologically [129]. EVs secreted by mesenchymal stromal cells (MSCs) have been proposed as important mechanistic relievers in response to cellular inflammation through paracrine effects [130-132]. In addition, Harting et al. demonstrated that EVs from MSCs (MSC-EVs) stimulated with TNF- $\alpha+$ IFN $-\gamma$ attenuated the release of proinflammatory cytokines in vitro [133]. Mao et al. proved the EVs derived from human MSCs can relieve the phenotypes of IBD in mice. After treatment with MSC-EVs in DSS-induced IBD mice, the expression of the IL-10 gene increased while those of the TNF- $\alpha$, IL-1b, IL-6, iNOS, and IL-7 genes decreased in the colon tissues [134]. Additionally, Yang et al. confirmed the potential of BMSC-EVs in protecting the TNBS-induced colitis model via attenuating oxidative stress and apoptosis [135]. Generally speaking, IBD is caused by the breakdown of innate immunity and the aberrant activation of the immune system. Therefore, it is consequently conceivable that EVs from the immune cells may be used as a new therapeutic intervention of IBD. As mentioned before, EVs derived from DCs can relieve the progress of disease via immune-stimulatory or immune-suppressive effects. Meanwhile, some conditioned DCs secreted EVs to make progress against the IBD [97]. The recent wave of research on EVs assists in the exploration of the utilization of artificial nanoparticles in disease treatment. A considerable amount of work has been performed regarding IBD treatment with EV-like nanoparticles. In 2019, Han et al. expanded the use of bioadhesive chitosan materials on colloidal-stable nanotherapeutics. This exhibited safe and precise accumulation to local diseased lesions in the gastrointestinal tract 
TABLE 1: Various source of EVs related to IBD.

\begin{tabular}{|c|c|c|c|}
\hline Source & Mechanism & Reference & \\
\hline \multirow{4}{*}{ Stem cell } & Alternating COX2/PGE2 pathway & [133] & MSC \\
\hline & Inhabiting iNOS and IL-7 pathway & [134] & MSC \\
\hline & Attenuating oxidative stress and apoptosis pathway & [135] & BMSC \\
\hline & Inhibiting EMT by targeting ZEB1 and ZEB2 & [129] & BMCS \\
\hline \multirow{4}{*}{ Milk } & Stimulate intestinal stem cell activity & [148] & Breast milk \\
\hline & Activating the hypoxia-inducible factor signaling pathway & [149] & Yak milk \\
\hline & Inhibiting P53 pathway & {$[150]$} & Porcine milk \\
\hline & Inhibiting oxidative stress pathway & {$[152]$} & Breast milk \\
\hline \multirow{4}{*}{ Immune cell } & Inhibiting Th1 cells proliferation and promoting Treg expansion & {$[102]$} & Myeloid-derived suppressor cells (MDSC) \\
\hline & $\mathrm{WNT} / \beta$-catenin signaling & {$[51,105]$} & Macrophage \\
\hline & Inducing Th1 polarized CD4+ T-cells & {$[93,94]$} & Dendritic cells \\
\hline & $\begin{array}{l}\text { Enhancing the inflammation response via } \\
\text { proinflammatory microRNAs and MPO }\end{array}$ & {$[98-101]$} & Neutrophil \\
\hline \multirow{4}{*}{ Microorganism } & Eliciting the release of proinflammatory IL- 8 & {$[51]$} & Escherichia coli C25 \\
\hline & Regulating $\mathrm{ZO}-1$ and $\mathrm{ZO}-2$ & [53] & Escherichia coli Nissle 1917 \\
\hline & Promoting intracellular $\mathrm{Ca}^{(2+)}$ signaling & {$[54]$} & Bacteroides thetaiotaomicron (BtMinpp) \\
\hline & Ameliorating the production of IL- 6 & {$[55]$} & Akkermansia muciniphila \\
\hline
\end{tabular}

COX2: cyclooxygenase 2; PGE2: prostaglandin E2; iNOS: inducible nitric oxide synthase; IL-7: interleukin 7; EMT: epithelial-mesenchymal transition; ZEB1: zinc finger E-box binding protein 1; Th1: T helper cell; Tregs: T regulatory cells; WNT: wingless/integrated; IL-8: interleukin 8; ZO-1: zonula occluden-1; IL-6: interleukin 6; MSC: mesenchymal stem cell; BMSC: bone mesenchymal stem cell; MDSC: myeloid-derived suppressor cells; BtMinpp: Bacteroides thetaiotaomicron.

[136]. In 2018, Zahra et al. utilized intestinal organoids as carriers of 5-ASA-loaded poly nanoparticles to alleviate IBD [137]. Similarly, Bo et al. used mannosylated bioreducible cationic polymers to synthesize RNA interference nanoparticles to reduce cytotoxicity and promote treatment effectiveness in IBD [138]. While innately derived from cells and microbiota, EVs are much more biocompatible and stable when compared with nanoparticles. What is more, EVs could also be engineered, thus indicating the therapeutic role in disease such as IBD [139, 140].

Breast milk not only is rich in nutrition but also provides a diverse array of microbiota and immunoglobulin. It may shape the neonate gut immune system actively and convert it toward a mature immune system capable of responding appropriately to encountered antigens [141-143]. EVs in milk are one of the most recently identified components that may influence intestinal homeostasis. Therefore, the discovery ten years ago that breast milk contains abundant immune modulatory EVs has earned plenty of attention in this field of study [144]. Additionally, breast milk EVs containing genetic material and proteins delivered to infant mucosae offer novel insights into the mechanisms of action for drug delivery in the intestinal tract. EVs are quite stable even in simulated gastric/pancreatic digestion [145] so that EV microRNAs in human breast milk can be delivered to the intestinal epithelia of infants [146]. Soon after, Liao et al. illustrated that milk-derived EVs enter human intestinal crypt-like cells, suggesting the possibility of EVs from breast milk altering the neonatal mucosal conditions [147]. Several studies have reported that treatment with milk EVs can significantly increase IEC viability, proliferation, and stem cell activity [148-150]. Breast milk reduces the incidence of necrotizing enterocolitis (NEC), and EVs in breast milk offer a new path in the mechanism for breast milk attenuating cell death in intestinal epithelial cells, as well as the possibility of transporting drugs in milk [151-154].

siRNA has a potential therapeutic effect but has various physiological limitations, including unstable delivery. Using lipofection to encapsulate AF488 in milk whey EVs guarantees their internalization by Caco- 2 cells [155]. Recently, protein within EVs has been the subject of intense research, and one such intestinal EV-containing molecule is TGF- $\beta 1$. Intestines produce EVs containing high levels of TGF- $\beta 1$ that can alleviate the severity of IBD by inducing regulatory T-cells and immunosuppressive dendritic cells in DSSinduced IBD mice [76]. Meanwhile, the endogenous molecule annexin A1 (ANXA1) has been reported to promote epithelial restitution in a colitis-induced mucosal damage model. In signaling via binging to formyl peptide receptors (FPRs), epithelial cells release the potent endogenous mediator ANXA1 as a component of EVs that promotes the repair of intestinal mucosal inflammation. Leoni et al. also observed the increased concentration of ANXA1 through EVs in the sera of patients with IBD and found that it correlated with disease severity [81]. Additionally, this correlation could conduce to EVs emerging as promising biomarkers not only to monitor IBD progression but also to have potential effects in future therapies. In fact, an in vivo proof of the study regarding ANXA1 proved that encapsulated targeted polymeric nanoparticles (Ac2-26 Col IV NPs) accelerated the recovery of intestinal inflammation in experimental IBD mice [81]. Similarly, nanoparticles, artificial EVs loaded with rifaximin, have high encapsulation efficiency, relatively high loading capacity, and a predetermined in vitro release profile 


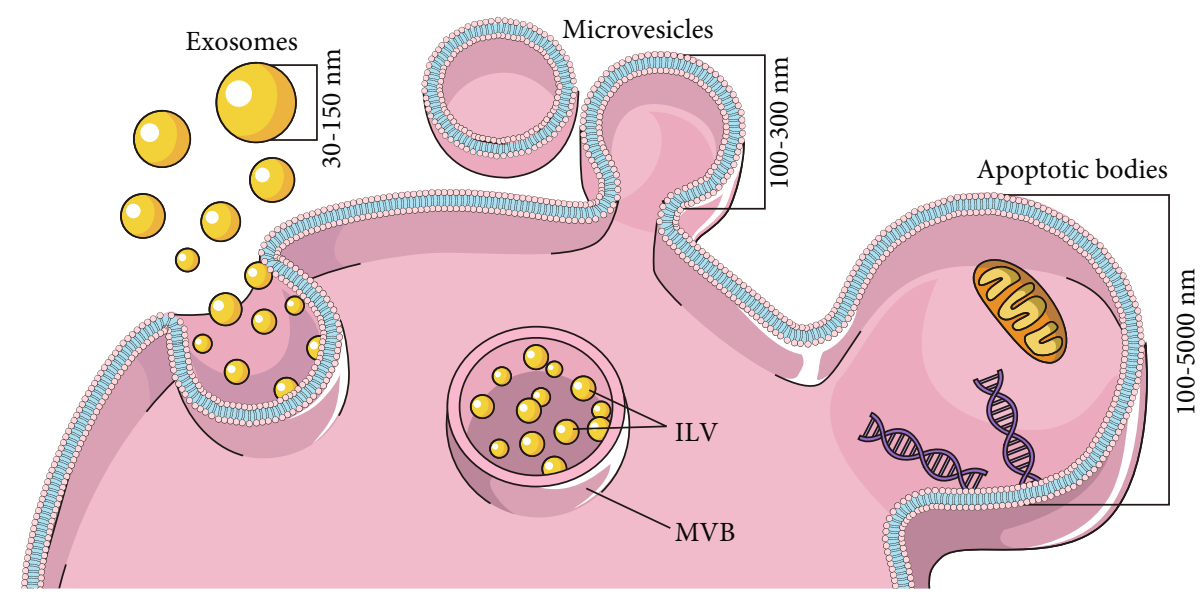

FIGURE 2: The interaction between bacteria, immune cells, and intestinal cells through EVs in gut. The schematic depicts the pathways by which OMVs derived from the member of microbiota take part in the hemostasis of intestines through various pathways. Bacteroides in virtue of HtrA packing in OMVs can facilitate its transmigration across polarized intestinal epithelial cells through the cleavage of the TJ and AJ. EVs from pathogenic bacteria can stimulate the production of interleukin-8 (IL-8) in IECs via the TLR5 and TLR4/MD-2 complex signaling pathway. OMVs from commensal bacteria containing peptidoglycans could colocalize with NOD1, trigger the NOD1 signaling pathway, and improve the expression of NF- $\kappa \mathrm{B}$, IL-6, and IL-8. EVs derived from bacteria on benefits of maintaining intestinal hemostasis reflected in increasing ZO-1 and claudin-14, decreasing claudin-2 in probiotic, and reducing the expression of IL-6 and TLR2dependent EV internalization by DCs. EVs from IECs carrying $\alpha \beta 6$ activate LTGF $\beta$ in intestinal tolerogenic DCs and Tregs. M $\phi$-derived EVs can enhance survival of enterocyte through WNT function. Note: OMVs: out membrane vesicles; HtrA: high-temperature requirement A; TJ: tight junction; AJ: adherens junctions; GJ: gap junction; TLR4/5: Toll-like receptor; IL-8: interleukin 8; IL-6: interleukin 6; $\mathrm{M} \phi$ : macrophages; EVs: extracellular vesicles; TGF- $\beta$ : transforming growth factor- $\beta$; NOD1: Nucleotide Binding Oligomerization Domain Containing 1; IECs: intestinal epithelial cells; LTGF $\beta$ : latent transforming growth factor- $\beta$; TGF- $\beta$ : transforming growth factor- $\beta$; DCs: dendritic cells; Tregs: T regulatory cells; WNT: wingless/integrated.

$[156,157]$. These studies regarding cargo manipulation suggest that EVs may be beneficial as drug delivery vehicles. So far, relevant EV studies considering practical clinical applications are usually preclinical studies based on animal or cell models. This indicates that further studies are required to explore the application prospects in clinical settings. However, such research is still in its infancy and should not be underestimated, whether in diagnosis or treatment.

\section{Conclusion}

In the current review, we discussed the source (Table 1), cargo, and origin of EVs and their roles in the pathogenesis and progression of IBD. We mainly focused on EVs from microbiota and enterocytes to clarify the relationships among EVs, microbiota, and intestinal inflammation (Figure 2). In addition, the clinical potential of EVs as biomarkers and their therapeutic effects on IBD were summarized.

\section{Conflicts of Interest}

The authors declare that the research was conducted in the absence of any commercial or financial relationship that could be constructed as a potential conflict of interest.

\section{Authors' Contributions}

XC, S-LW, S-BZ, Y-HS, and PP retrieved and analyzed concerned literatures. XC, S-LW, and Y-HS wrote the manu- script. LG designed the table and figures. JY, Z-SL, and YB revised the manuscript. All the authors agreed to be accountable for the content of the work. Xin Chang, Shu-Ling Wang, Sheng-Bing Zhao, Yi-Hai Shi, and Peng Pan contributed equally to this work.

\section{Acknowledgments}

Dr. Bai Yu is supported by the National Key R\&D Program of China (2017YFC1308800, 2018YFC1313103), National Natural Science Foundation of China (Grant Nos. 81670473 and 81873546), "Shu Guang" project of Shanghai Municipal Education Commission and Shanghai Education Development Foundation (No. 19SG30), and Three Engineering Training Funds in Shenzhen (No. SYLY201718). Dr. Jun Yao is supported by the National Natural Science Foundation of China (grant no. 81800489), Three Engineering Training Funds in Shenzhen (no. SYLY201718), and Technical Research and Development Project of Shenzhen (nos. JCYJ20150403101028164 and JCYJ20170307100538697). Dr. Hai Shi-Yi is supported by the Science and Technology Development Fund of Shanghai Pudong New Area (Grant No. PKJ2016-Y15).

\section{References}

[1] L. W. Peterson and D. Artis, "Intestinal epithelial cells: regulators of barrier function and immune homeostasis," Nature Reviews Immunology, vol. 14, no. 3, pp. 141-153, 2014. 
[2] R. Goll and G. A. van Beelen, "Intestinal barrier homeostasis in inflammatory bowel disease," Scandinavian Journal of Gastroenterology, vol. 50, no. 1, pp. 3-12, 2015.

[3] K. J. Maloy and F. Powrie, "Intestinal homeostasis and its breakdown in inflammatory bowel disease," Nature, vol. 474, no. 7351, pp. 298-306, 2011.

[4] E. I. Buzas, B. Gyorgy, G. Nagy, A. Falus, and S. Gay, "Emerging role of extracellular vesicles in inflammatory diseases," Nature Reviews Rheumatology, vol. 10, no. 6, pp. 356-364, 2014.

[5] A. Kulp and M. J. Kuehn, "Biological functions and biogenesis of secreted bacterial outer membrane vesicles," Annual Review of Microbiology, vol. 64, pp. 163-184, 2010.

[6] E. D. Avila-Calderon, M. G. Araiza-Villanueva, J. C. Cancino-Diaz et al., "Roles of bacterial membrane vesicles," Archives of Microbiology, vol. 197, no. 1, pp. 1-10, 2015.

[7] R. B. Sartor, "Microbial influences in inflammatory bowel diseases," Gastroenterology, vol. 134, no. 2, pp. 577-594, 2008.

[8] F. Fava and S. Danese, "Intestinal microbiota in inflammatory bowel disease: friend of foe?," World Journal of Gastroenterology, vol. 17, no. 5, pp. 557-566, 2011.

[9] F. Sommer, J. M. Anderson, R. Bharti, J. Raes, and P. Rosenstiel, "The resilience of the intestinal microbiota influences health and disease," Nature Reviews Microbiology, vol. 15, no. 10, pp. 630-638, 2017.

[10] M. Toyofuku, N. Nomura, and L. Eberl, "Types and origins of bacterial membrane vesicles," Nature Reviews Microbiology, vol. 17, no. 1, pp. 13-24, 2019.

[11] J. W. Choi, J. H. Um, J. H. Cho, and H. J. Lee, "Tiny RNAs and their voyage via extracellular vesicles: secretion of bacterial small RNA and eukaryotic microRNA," Experimental biology and medicine, vol. 242, no. 15, pp. 1475-1481, 2017.

[12] E. Schulz, A. Goes, R. Garcia et al., "Biocompatible bacteriaderived vesicles show inherent antimicrobial activity," Journal of Controlled Release: Official Journal of the Controlled Release Society, vol. 290, pp. 46-55, 2018.

[13] M. Colombo, G. Raposo, and C. Thery, "Biogenesis, secretion, and intercellular interactions of exosomes and other extracellular vesicles," Annual Review of Cell and Developmental Biology, vol. 30, pp. 255-289, 2014.

[14] J. Lotvall, A. F. Hill, F. Hochberg et al., "Minimal experimental requirements for definition of extracellular vesicles and their functions: a position statement from the International Society for Extracellular Vesicles," Journal of extracellular vesicles, vol. 3, no. 1, article 26913, 2014.

[15] M. F. Haurat, W. Elhenawy, and M. F. Feldman, "Prokaryotic membrane vesicles: new insights on biogenesis and biological roles," Biological Chemistry, vol. 396, no. 2, pp. 95-109, 2015.

[16] S. B. Gould, S. G. Garg, and W. F. Martin, "Bacterial vesicle secretion and the evolutionary origin of the eukaryotic endomembrane system," Trends in Microbiology, vol. 24, no. 7, pp. 525-534, 2016.

[17] M. L. Merchant, I. M. Rood, J. K. J. Deegens, and J. B. Klein, "Isolation and characterization of urinary extracellular vesicles: implications for biomarker discovery," Nature Reviews Nephrology, vol. 13, no. 12, pp. 731-749, 2017.

[18] V. Severino, J. M. Dumonceau, M. Delhaye et al., "Extracellular vesicles in bile as markers of malignant biliary stenoses," Gastroenterology, vol. 153, no. 2, pp. 495-504.e8, 2017.
[19] M. Mathieu, L. Martin-Jaular, G. Lavieu, and C. Théry, "Specificities of secretion and uptake of exosomes and other extracellular vesicles for cell-to-cell communication," Nature Cell Biology, vol. 21, no. 1, pp. 9-17, 2019.

[20] M. Fleshner and C. R. Crane, "Exosomes, DAMPs and miRNA: features of stress physiology and immune homeostasis," Trends in Immunology, vol. 38, no. 10, pp. 768-776, 2017.

[21] M. Schmid and T. H. Jensen, "The exosome: a multipurpose RNA-decay machine," Trends in Biochemical Sciences, vol. 33, no. 10, pp. 501-510, 2008.

[22] E. van der Pol, A. N. Böing, P. Harrison, A. Sturk, and R. Nieuwland, "Classification, functions, and clinical relevance of extracellular vesicles," Pharmacological Reviews, vol. 64, no. 3, pp. 676-705, 2012.

[23] P. D. Singorenko, V. Chang, A. Whitcombe et al., "Isolation of membrane vesicles from prokaryotes: a technical and biological comparison reveals heterogeneity," Journal of extracellular vesicles, vol. 6, no. 1, article 1324731, 2017.

[24] V. Muralidharan-Chari, J. Clancy, C. Plou et al., "ARF6-regulated shedding of tumor cell-derived plasma membrane microvesicles," Current Biology, vol. 19, no. 22, pp. 18751885, 2009.

[25] R. Xu, D. W. Greening, A. Rai, H. Ji, and R. J. Simpson, "Highly-purified exosomes and shed microvesicles isolated from the human colon cancer cell line LIM1863 by sequential centrifugal ultrafiltration are biochemically and functionally distinct," Methods, vol. 87, pp. 11-25, 2015.

[26] H. A. Bladen and J. F. Waters, "Electron microscopic study of some strains of bacteroides," Journal of Bacteriology, vol. 86, pp. 1339-1344, 1963.

[27] E. Y. Lee, D. Y. Choi, D. K. Kim et al., "Gram-positive bacteria produce membrane vesicles: proteomics-based characterization of Staphylococcus aureus-derived membrane vesicles," Proteomics, vol. 9, no. 24, pp. 5425-5436, 2009.

[28] L. Brown, J. M. Wolf, R. Prados-Rosales, and A. Casadevall, "Through the wall: extracellular vesicles in Gram-positive bacteria, mycobacteria and fungi," Nature Reviews Microbiology, vol. 13, no. 10, pp. 620-630, 2015.

[29] J. H. Kim, J. Lee, J. Park, and Y. S. Gho, "Gram-negative and Gram-positive bacterial extracellular vesicles," Seminars in Cell \& Developmental Biology, vol. 40, pp. 97-104, 2015.

[30] A. A. Farooqi, N. N. Desai, M. Z. Qureshi et al., "Exosome biogenesis, bioactivities and functions as new delivery systems of natural compounds," Biotechnology Advances, vol. 36, no. 1, pp. 328-334, 2018.

[31] M. P. Bebelman, M. J. Smit, D. M. Pegtel, and S. R. Baglio, "Biogenesis and function of extracellular vesicles in cancer," Pharmacology \& Therapeutics, vol. 188, pp. 1-11, 2018.

[32] S. L. N. Maas, X. O. Breakefield, and A. M. Weaver, "Extracellular vesicles: unique intercellular delivery vehicles," Trends in Cell Biology, vol. 27, no. 3, pp. 172-188, 2017.

[33] V. Agrahari, V. Agrahari, P. A. Burnouf, C. H. Chew, and T. Burnouf, "Extracellular microvesicles as new industrial therapeutic frontiers," Trends in Biotechnology, vol. 37, no. 7, pp. 707-729, 2019.

[34] M. Simons and G. Raposo, "Exosomes - vesicular carriers for intercellular communication," Current Opinion in Cell Biology, vol. 21, no. 4, pp. 575-581, 2009.

[35] J. Kowal, M. Tkach, and C. Théry, "Biogenesis and secretion of exosomes," Current Opinion in Cell Biology, vol. 29, pp. 116-125, 2014. 
[36] B. L. Deatherage and B. T. Cookson, "Membrane vesicle release in bacteria, eukaryotes, and archaea: a conserved yet underappreciated aspect of microbial life," Infection and Immunity, vol. 80, no. 6, pp. 1948-1957, 2012.

[37] T. C. Liu and T. S. Stappenbeck, "Genetics and pathogenesis of inflammatory bowel disease," Annual Review of Pathology: Mechanisms of Disease, vol. 11, no. 1, pp. 127-148, 2016.

[38] D. Gevers, S. Kugathasan, L. A. Denson et al., "The Treatment-Naive Microbiome in New-Onset Crohn's Disease," Cell Host \& Microbe, vol. 15, no. 3, pp. 382-392, 2014.

[39] K. Koeppen, T. H. Hampton, M. Jarek et al., "A novel mechanism of host-pathogen interaction through sRNA in bacterial outer membrane vesicles," PLoS Pathogens, vol. 12, no. 6, article e1005672, 2016.

[40] V. B. Jeroen, A. D. Kraneveld, R. Lieke, K. Nienke, G. Johan, and A. P. Vos, "Extracellular vesicles modulate host-microbe responses by altering TLR2 activity and phagocytosis," PLoS One, vol. 9, no. 2, article e89121, 2014.

[41] S. N. Chatterjee and J. Das, "Electron microscopic observations on the excretion of cell-wall material by Vibrio cholerae," Journal of General Microbiology, vol. 49, no. 1, pp. 1-11, 1967.

[42] S. Ismail, M. B. Hampton, and J. I. Keenan, "Helicobacter pylori outer membrane vesicles modulate proliferation and interleukin- 8 production by gastric epithelial cells," Infection and Immunity, vol. 71, no. 10, pp. 5670-5675, 2003.

[43] M. Bielaszewska, M. Marejkova, A. Bauwens, L. KunsmannProkscha, A. Mellmann, and H. Karch, "Enterohemorrhagic Escherichia coli O157 outer membrane vesicles induce interleukin 8 production in human intestinal epithelial cells by signaling via toll-like receptors TLR4 and TLR5 and activation of the nuclear factor NF- $\kappa \mathrm{B}$," International journal of medical microbiology: IJMM, vol. 308, no. 7, pp. 882-889, 2018.

[44] M. Bielaszewska, C. Ruter, L. Kunsmann et al., "Enterohemorrhagic Escherichia coli hemolysin employs outer membrane vesicles to target mitochondria and cause endothelial and epithelial apoptosis," PLoS Pathogens, vol. 9, no. 12, article e1003797, 2013.

[45] M. A. Canas, M. J. Fabrega, R. Gimenez, J. Badia, and L. Baldoma, "Outer membrane vesicles from probiotic and commensal Escherichia coli activate NOD1-mediated immune responses in intestinal epithelial cells," Frontiers in Microbiology, vol. 9, p. 498, 2018.

[46] M. A. Canas, R. Gimenez, M. J. Fabrega, L. Toloza, L. Baldoma, and J. Badia, "Outer membrane vesicles from the probiotic Escherichia coli Nissle 1917 and the commensal ECOR12 enter intestinal epithelial cells via clathrin-dependent endocytosis and elicit differential effects on DNA damage," PLoS One, vol. 11, no. 8, article e0160374, 2016.

[47] X. J. Gao, T. Li, B. Wei et al., "Bacterial outer membrane vesicles from dextran sulfate sodium-induced colitis differentially regulate intestinal UDP-glucuronosyltransferase $1 \mathrm{~A} 1$ partially through toll-like receptor 4/mitogen-activated protein kinase/phosphatidylinositol 3-kinase pathway," Drug Metabolism and Disposition: The Biological Fate of Chemicals, vol. 46, no. 3, pp. 292-302, 2018.

[48] A. Mondal, R. Tapader, N. S. Chatterjee et al., "Cytotoxic and inflammatory responses induced by outer membrane vesicle-associated biologically active proteases from Vibrio cholerae," Infection and Immunity, vol. 84, no. 5, pp. 14781490, 2016.
[49] D. Chatterjee and K. Chaudhuri, "Association of cholera toxin with Vibrio cholerae outer membrane vesicles which are internalized by human intestinal epithelial cells," FEBS Letters, vol. 585, no. 9, pp. 1357-1362, 2011.

[50] D. Chatterjee and K. Chaudhuri, "Vibrio cholerae O395 outer membrane vesicles modulate intestinal epithelial cells in a NOD1 protein-dependent manner and induce dendritic cell-mediated Th2/Th17 cell responses," The Journal of Biological Chemistry, vol. 288, no. 6, pp. 4299-4309, 2013.

[51] D. A. Patten, E. Hussein, S. P. Davies, P. N. Humphreys, and A. Collett, "Commensal-derived OMVs elicit a mild proinflammatory response in intestinal epithelial cells," Microbiology, vol. 163, no. 5, pp. 702-711, 2017.

[52] I. Trebichavsky, I. Splichal, V. Rada, and A. Splichalova, "Modulation of natural immunity in the gut by Escherichia coli strain Nissle 1917," Nutrition Reviews, vol. 68, no. 8, pp. 459-464, 2010.

[53] C. S. Alvarez, J. Badia, M. Bosch, R. Gimenez, and L. Baldoma, "Outer membrane vesicles and soluble factors released by probiotic Escherichia coli Nissle 1917 and commensal ECOR63 enhance barrier function by regulating expression of tight junction proteins in intestinal epithelial cells," Frontiers in Microbiology, vol. 7, p. 1981, 2016.

[54] R. Stentz, S. Osborne, N. Horn et al., “A bacterial homolog of a eukaryotic inositol phosphate signaling enzyme mediates cross-kingdom dialog in the mammalian gut," Cell Reports, vol. 6, no. 4, pp. 646-656, 2014.

[55] C. S. Kang, M. Ban, E. J. Choi et al., "Extracellular vesicles derived from gut microbiota, especially Akkermansia muciniphila, protect the progression of dextran sulfate sodiuminduced colitis," PLoS One, vol. 8, no. 10, article e76520, 2013.

[56] M. Boehm, D. Simson, U. Escher et al., "Function of serine protease HtrA in the lifecycle of the foodborne pathogen Campylobacter jejuni," European journal of microbiology \& immunology, vol. 8, no. 3, pp. 70-77, 2018.

[57] A. Elmi, F. Nasher, H. Jagatia et al., "Campylobacter jejuni outer membrane vesicle-associated proteolytic activity promotes bacterial invasion by mediating cleavage of intestinal epithelial cell E-cadherin and occludin," Cellular Microbiology, vol. 18, no. 4, pp. 561-572, 2016.

[58] J. L. Coleman, A. Toledo, and J. L. Benach, "HtrA of Borrelia burgdorferi leads to decreased swarm motility and decreased production of pyruvate," mBio, vol. 9, no. 4, 2018.

[59] P. X. Medina Rangel, E. Moroni, F. Merlier et al., "Chemical antibody mimics inhibit cadherin-mediated cell-cell adhesion: a promising strategy for cancer therapy," Angewandte Chemie International Edition, 2019.

[60] T. D. Godwin, S. T. Kelly, T. P. Brew et al., "E-cadherin-deficient cells have synthetic lethal vulnerabilities in plasma membrane organisation, dynamics and function," Gastric Cancer, vol. 22, no. 2, pp. 273-286, 2019.

[61] C. M. Abfalter, M. Schubert, C. Götz, T. P. Schmidt, G. Posselt, and S. Wessler, "HtrA-mediated E-cadherin cleavage is limited to DegP and DegQ homologs expressed by gram-negative pathogens," Cell Communication and Signaling, vol. 14, no. 1, p. 30, 2016.

[62] A. Elmi, E. Watson, P. Sandu et al., "Campylobacter jejuni outer membrane vesicles play an important role in bacterial interactions with human intestinal epithelial cells," Infection and Immunity, vol. 80, no. 12, pp. 4089-4098, 2012. 
[63] M. P. Jeffrey, J. L. Strap, H. J. Taggart, and J. M. Green-Johnson, "Suppression of Intestinal Epithelial Cell Chemokine Production by Lactobacillus rhamnosus R0011 and Lactobacillus helveticus R0389 Is Mediated by Secreted Bioactive Molecules," Frontiers in Immunology, vol. 9, article 2639, 2018.

[64] T. Zheng, B. Zhang, C. Chen et al., "Protein kinase p38 $\alpha$ signaling in dendritic cells regulates colon inflammation and tumorigenesis," Proceedings of the National Academy of Sciences, vol. 115, no. 52, pp. E12313-E12322, 2018.

[65] B. L. Kelsall and F. Leon, "Involvement of intestinal dendritic cells in oral tolerance, immunity to pathogens, and inflammatory bowel disease," Immunological Reviews, vol. 206, no. 1, pp. 132-148, 2005.

[66] A. E. Morelli, A. T. Larregina, W. J. Shufesky et al., "Endocytosis, intracellular sorting, and processing of exosomes by dendritic cells," Blood, vol. 104, no. 10, pp. 3257-3266, 2004.

[67] X. P. Lin, N. Almqvist, and E. Telemo, "Human small intestinal epithelial cells constitutively express the key elements for antigen processing and the production of exosomes," Blood Cells, Molecules \& Diseases, vol. 35, no. 2, pp. 122-128, 2005.

[68] J. Mallegol, G. Van Niel, C. Lebreton et al., “T84-intestinal epithelial exosomes bear MHC class II/peptide complexes potentiating antigen presentation by dendritic cells," Gastroenterology, vol. 132, no. 5, pp. 1866-1876, 2007.

[69] G. Van Niel, G. Raposo, C. Candalh et al., "Intestinal epithelial cells secrete exosome-like vesicles," Gastroenterology, vol. 121, no. 2, pp. 337-349, 2001.

[70] J. Mallegol, G. van Niel, and M. Heyman, "Phenotypic and functional characterization of intestinal epithelial exosomes," Blood Cells, Molecules \& Diseases, vol. 35, no. 1, pp. 11-16, 2005.

[71] J. Merkenschlager, U. Eksmond, L. Danelli et al., "MHC class II cell-autonomously regulates self-renewal and differentiation of normal and malignant B cells," Blood, vol. 133, no. 10, pp. 1108-1118, 2019.

[72] L. Mari, S. J. M. Hoefnagel, D. Zito et al., "microRNA 125a regulates MHC-I expression on esophageal adenocarcinoma cells, associated with suppression of antitumor immune response and poor outcomes of patients," Gastroenterology, vol. 155, no. 3, pp. 784-798, 2018.

[73] V. G. Niel, "Intestinal epithelial exosomes carry MHC class II/peptides able to inform the immune system in mice," Gut, vol. 52, no. 12, pp. 1690-1697, 2003.

[74] C. Xiao, S. Chun-Hua, F. Bai-Sui et al., "Intestinal epithelial cell-derived integrin $\alpha \beta 6$ plays an important role in the induction of regulatory $\mathrm{T}$ cells and inhibits an antigenspecific Th2 response," Journal of Leukocyte Biology, vol. 90, no. 4, pp. 751-759, 2011.

[75] M. Karlsson, S. Lundin, U. Dahlgren, H. Kahu, I. Pettersson, and E. Telemo, "'Tolerosomes" are produced by intestinal epithelial cells," European Journal of Immunology, vol. 31, no. 10, pp. 2892-2900, 2001.

[76] L. Jiang, Y. Shen, D. Guo et al., "EpCAM-dependent extracellular vesicles from intestinal epithelial cells maintain intestinal tract immune balance," Nature Communications, vol. 7, no. 1, article 13045, 2016.

[77] M. Kojima, T. W. Costantini, B. P. Eliceiri, T. W. Chan, A. Baird, and R. Coimbra, "Gut epithelial cell-derived exosomes trigger post-trauma immune dysfunction," Journal of
Trauma and Acute Care Surgery, vol. 84, no. 2, pp. 257264, 2017.

[78] L. Meyaard, A. R. van der Vuurst de Vries, T. de Ruiter, L. L. Lanier, J. H. Phillips, and H. Clevers, "The epithelial cellular adhesion molecule (Ep-CAM) is a ligand for the leukocyteassociated immunoglobulin-like receptor (LAIR)," The Journal of Experimental Medicine, vol. 194, no. 1, pp. 107-112, 2001.

[79] L. Giovanna, A. Ashfaqul, N. Philipp-Alexander et al., "Annexin A1, formyl peptide receptor, and NOX1 orchestrate epithelial repair," Journal of Clinical Investigation, vol. 123, no. 1, pp. 443-454, 2013.

[80] M. Perretti and F. D'Acquisto, "Annexin A1 and glucocorticoids as effectors of the resolution of inflammation," Nature Reviews Immunology, vol. 9, no. 1, pp. 62-70, 2009.

[81] G. Leoni, P. A. Neumann, N. Kamaly et al., "Annexin A1containing extracellular vesicles and polymeric nanoparticles promote epithelial wound repair," The Journal of Clinical Investigation, vol. 125, no. 3, pp. 1215-1227, 2015.

[82] X. Zhang, S. A. Deeke, Z. Ning et al., "Metaproteomics reveals associations between microbiome and intestinal extracellular vesicle proteins in pediatric inflammatory bowel disease," Nature Communications, vol. 9, no. 1, p. 2873, 2018.

[83] E. N. D. Palladino, C. L. Hartman, and C. J. Albert, "The chlorinated lipidome originating from myeloperoxidase-derived $\mathrm{HOCl}$ targeting plasmalogens: Metabolism, clearance, and biological properties," Archives of Biochemistry and Biophysics, vol. 641, pp. 31-38, 2018.

[84] M. J. Davies, "Myeloperoxidase-derived oxidation: mechanisms of biological damage and its prevention," Journal of Clinical Biochemistry and Nutrition, vol. 48, no. 1, pp. 8-19, 2011.

[85] E. Malle, G. Marsche, J. Arnhold, and M. J. Davies, "Modification of low-density lipoprotein by myeloperoxidasederived oxidants and reagent hypochlorous acid," Biochimica et Biophysica Acta (BBA) - Molecular and Cell Biology of Lipids, vol. 1761, no. 4, pp. 392-415, 1761.

[86] S. Cuzzocrea, E. Mazzon, I. Serraino et al., "Melatonin reduces dinitrobenzene sulfonic acid-induced colitis," Journal of Pineal Research, vol. 30, no. 1, pp. 1-12, 2001.

[87] S. Mitsuhashi, L. Feldbrugge, E. Csizmadia, M. Mitsuhashi, S. C. Robson, and A. C. Moss, "Luminal extracellular vesicles $(\mathrm{EVs})$ in inflammatory bowel disease (IBD) exhibit proinflammatory effects on epithelial cells and macrophages," Inflammatory Bowel Diseases, vol. 22, no. 7, pp. 1587-1595, 2016.

[88] Z. Deng, J. Mu, M. Tseng et al., "Enterobacteria-secreted particles induce production of exosome-like S1P-containing particles by intestinal epithelium to drive Th17-mediated tumorigenesis," Nature Communications, vol. 6, no. 1, p. 6956, 2015.

[89] G. T. Kunkel, M. Maceyka, S. Milstien, and S. Spiegel, "Targeting the sphingosine-1-phosphate axis in cancer, inflammation and beyond," Nature Reviews Drug Discovery, vol. 12, no. 9, pp. 688-702, 2013.

[90] N. C. Hait and A. Maiti, "The role of sphingosine-1phosphate and ceramide-1-phosphate in inflammation and cancer," Mediators of Inflammation, vol. 2017, Article ID 4806541, 17 pages, 2017.

[91] S. N. Patmanathan, W. Wang, L. F. Yap, D. R. Herr, and I. C. Paterson, "Mechanisms of sphingosine 1-phosphate receptor 
signalling in cancer," Cellular Signalling, vol. 34, pp. 66-75, 2017.

[92] M. F. S. Lindenbergh and W. Stoorvogel, “Antigen presentation by extracellular vesicles from professional antigenpresenting cells," Annual Review of Immunology, vol. 36, no. 1, pp. 435-459, 2018.

[93] S. H. Kim, N. R. Bianco, W. J. Shufesky, A. E. Morelli, and P. D. Robbins, "Effective treatment of inflammatory disease models with exosomes derived from dendritic cells genetically modified to express IL-4," Journal of immunology, vol. 179, no. 4, pp. 2242-2249, 2007.

[94] M. Tkach, J. Kowal, A. E. Zucchetti et al., "Qualitative differences in T-cell activation by dendritic cell-derived extracellular vesicle subtypes," The EMBO Journal, vol. 36, no. 20, pp. 3012-3028, 2017.

[95] Z. Cai, W. Zhang, F. Yang et al., "Immunosuppressive exosomes from TGF- $\beta 1$ gene-modified dendritic cells attenuate Th17-mediated inflammatory autoimmune disease by inducing regulatory T cells," Cell Research, vol. 22, no. 3, pp. 607610, 2012.

[96] X. Yang, S. Meng, H. Jiang, T. Chen, and W. Wu, "Exosomes derived from interleukin-10-treated dendritic cells can inhibit trinitrobenzene sulfonic acid-induced rat colitis," Scandinavian Journal of Gastroenterology, vol. 45, no. 10, pp. 1168-1177, 2010.

[97] L. Wang, Z. Yu, S. Wan et al., "exosomes derived from dendritic cells treated with Schistosoma japonicum soluble egg antigen attenuate DSS-induced colitis," Frontiers in Pharmacology, vol. 8, p. 651, 2017.

[98] T. W. Slater, A. Finkielsztein, L. A. Mascarenhas, L. C. Mehl, V. Butin-Israeli, and R. Sumagin, "Neutrophil microparticles deliver active myeloperoxidase to injured mucosa to inhibit epithelial wound healing," The Journal of immunology, vol. 198, no. 7, pp. 2886-2897, 2017.

[99] V. Butin-Israeli, T. M. Bui, H. L. Wiesolek et al., "Neutrophilinduced genomic instability impedes resolution of inflammation and wound healing," Journal of Clinical Investigation, vol. 129, no. 2, pp. 712-726, 2019.

[100] T. M. Bui and R. Sumagin, "Progressing from recurring tissue injury to genomic instability: a new mechanism of neutrophil pathogenesis," DNA and Cell Biology, vol. 38, no. 8, pp. 747753, 2019.

[101] V. Butin-Israeli, M. C. Houser, M. Feng et al., "Deposition of microparticles by neutrophils onto inflamed epithelium: a new mechanism to disrupt epithelial intercellular adhesions and promote transepithelial migration," The FASEB journal, vol. 30, no. 12, pp. 4007-4020, 2016.

[102] Y. Wang, J. Tian, X. Tang et al., "Exosomes released by granulocytic myeloid-derived suppressor cells attenuate DSS-induced colitis in mice," Oncotarget, vol. 7, no. 13, pp. 15356-15368, 2016.

[103] A. Gregorieff, D. Pinto, H. Begthel, O. Destree, M. Kielman, and H. Clevers, "Expression pattern of Wnt signaling components in the adult intestine," Gastroenterology, vol. 129, no. 2, pp. 626-638, 2005.

[104] F. Kuhnert, C. R. Davis, H. T. Wang et al., "Essential requirement for Wnt signaling in proliferation of adult small intestine and colon revealed by adenoviral expression of Dickkopf-1," Proceedings of the National Academy of Sciences of the United States of America, vol. 101, no. 1, pp. 266-271, 2004.
[105] S. Saha, E. Aranda, Y. Hayakawa et al., "Macrophage-derived extracellular vesicle-packaged WNTs rescue intestinal stem cells and enhance survival after radiation injury," Nature Communications, vol. 7, no. 1, article 13096, 2016.

[106] L. Ling, D. Masica, M. Ishida et al., "Human bile contains Micro RNA-laden extracellular vesicles that can be used for cholangiocarcinoma diagnosis," vol. 60, 2014.

[107] R. J. Lobb, M. Becker, S. Wen Wen et al., "Optimized exosome isolation protocol for cell culture supernatant and human plasma," Journal of Extracellular Vesicles, vol. 4, no. 1, article 27031, 2015.

[108] M. L. Alvarez, M. Khosroheidari, R. Kanchi Ravi, and J. K. DiStefano, "Comparison of protein, microRNA, and mRNA yields using different methods of urinary exosome isolation for the discovery of kidney disease biomarkers," Kidney International, vol. 82, no. 9, pp. 1024-1032, 2012.

[109] C. Théry, S. Amigorena, G. Raposo, and A. Clayton, "Isolation and characterization of exosomes from cell culture supernatants and biological fluids," Current protocols in cell biology, vol. 30, no. 1, pp. 3.22.1-3.22.29, 2006.

[110] Y. Tian, L. Ma, M. Gong et al., "Protein profiling and sizing of extracellular vesicles from colorectal cancer patients via flow cytometry," ACS Nano, vol. 12, no. 1, pp. 671-680, 2018.

[111] T. Matsumura, K. Sugimachi, H. Iinuma et al., "Exosomal microRNA in serum is a novel biomarker of recurrence in human colorectal cancer," British Journal of Cancer, vol. 113, no. 2, pp. 275-281, 2015.

[112] B. J. Kim, S. K. Yang, J. S. Kim et al., "Trends of ulcerative colitis-associated colorectal cancer in Korea: a KASID study," Journal of Gastroenterology and Hepatology, vol. 24, no. 4, pp. 667-671, 2009.

[113] Y. S. Jung, M. Han, S. Park, W. H. Kim, and J. H. Cheon, "Cancer risk in the early stages of inflammatory bowel disease in Korean patients: a nationwide population-based study," Journal of Crohn's and Colitis, vol. 11, no. 8, pp. 954-962, 2017.

[114] R. D. Bojesen, L. B. Riis, E. Høgdall, O. H. Nielsen, and T. Jess, "Inflammatory bowel disease and small bowel cancer risk, clinical characteristics, and histopathology: a populationbased study," Clinical gastroenterology and hepatology, vol. 15, no. 12, pp. 1900-1907.e2, 2017.

[115] C. Polytarchou, D. W. Hommes, T. Palumbo et al., "MicroRNA214 Is Associated With Progression of Ulcerative Colitis, and Inhibition Reduces Development of Colitis and Colitis-Associated Cancer in Mice," Gastroenterology, vol. 149, no. 4, pp. 981-92.e11, 2015.

[116] P. L. Di Wu, X. Mi, and J. Miao, "Exosomal miR-214 from endometrial stromal cells inhibits endometriosis fibrosis," Molecular Human Reproduction, vol. 24, no. 7, pp. 357-365, 2018.

[117] C. Barbagallo, D. Brex, A. Caponnetto et al., "LncRNA UCA1, upregulated in CRC biopsies and downregulated in serum exosomes, controls mRNA expression by RNA-RNA interactions," Molecular therapy-Nucleic acids, vol. 12, pp. 229-241, 2018.

[118] V. Köberle, T. Pleli, C. Schmithals et al., "Differential stability of cell-free circulating microRNAs: implications for their utilization as biomarkers," PLoS One, vol. 8, no. 9, article e75184, 2013.

[119] S. Gurunathan, M.-H. Kang, M. Jeyaraj, M. Qasim, and J.H. Kim, "Review of the isolation, characterization, biological 
function, and multifarious therapeutic approaches of exosomes," Cells, vol. 8, no. 4, p. 307, 2019.

[120] M. Wang, F. Yu, H. Ding, Y. Wang, P. Li, and K. Wang, "Emerging Function and Clinical Values of Exosomal MicroRNAs in Cancer," Molecular therapy-Nucleic acids, vol. 16, pp. 791-804, 2019.

[121] X. Zheng, F. Chen, Q. Zhang et al., "Salivary exosomal PSMA7: a promising biomarker of inflammatory bowel disease," Protein \& Cell, vol. 8, no. 9, pp. 686-695, 2017.

[122] Y. Jia, T. Song, C. Wei et al., "Negative regulation of MAVSmediated innate immune response by PSMA7," The Journal of Immunology, vol. 183, no. 7, pp. 4241-4248, 2009.

[123] A. Minelli, C. Magri, A. Barbon et al., "Proteasome system dysregulation and treatment resistance mechanisms in major depressive disorder," Translational Psychiatry, vol. 5, no. 12, p. e687, 2015.

[124] X. F. Ruan, C. W. Ju, Y. Shen et al., "Suxiao Jiuxin pill promotes exosome secretion from mouse cardiac mesenchymal stem cells in vitro," Acta Pharmacologica Sinica, vol. 39, no. 4, pp. 569-578, 2018.

[125] M. Ostrowski, N. B. Carmo, S. Krumeich et al., "Rab27a and Rab27b control different steps of the exosome secretion pathway," Nature cell biology, vol. 12, no. 1, pp. 19-30, 2010.

[126] A. T. Xu, J. T. Lu, Z. H. Ran, and Q. Zheng, "Exosome in intestinal mucosal immunity," Journal of Gastroenterology and Hepatology, vol. 31, no. 10, pp. 1694-1699, 2016.

[127] M. Alexander, A. G. Ramstead, K. M. Bauer et al., "Rab27dependent exosome production inhibits chronic inflammation and enables acute responses to inflammatory stimuli," Journal of immunology, vol. 199, no. 10, pp. 3559-3570, 2017.

[128] I. S. Okoye, S. M. Coomes, V. S. Pelly et al., "MicroRNA-Containing T-Regulatory-Cell-Derived Exosomes Suppress Pathogenic T Helper 1 Cells," Immunity, vol. 41, no. 3, p. 503, 2014.

[129] J. Yang, C. Z. Zhou, R. Zhu et al., "miR-200b-containing microvesicles attenuate experimental colitis associated intestinal fibrosis by inhibiting epithelial-mesenchymal transition," Journal of Gastroenterology and Hepatology, vol. 32, no. 12, pp. 1966-1974, 2017.

[130] A. A. Nargesi, L. O. Lerman, and A. Eirin, "Mesenchymal stem cell-derived extracellular vesicles for renal repair," Current Gene Therapy, vol. 17, no. 1, pp. 29-42, 2017.

[131] M. L. Stone, Y. Zhao, J. Robert Smith et al., "Mesenchymal stromal cell-derived extracellular vesicles attenuate lung ischemia-reperfusion injury and enhance reconditioning of donor lungs after circulatory death," Respiratory Research, vol. 18, no. 1, p. 212, 2017.

[132] X. D. Tang, L. Shi, A. Monsel et al., "Mesenchymal stem cell microvesicles attenuate acute lung injury in mice partly mediated by Ang-1 mRNA," Stem cells, vol. 35, no. 7, pp. 1849$1859,2017$.

[133] M. T. Harting, A. K. Srivastava, S. Zhaorigetu et al., "Inflammation-stimulated mesenchymal stromal cell-derived extracellular vesicles attenuate inflammation," Stem cells, vol. 36, no. 1, pp. 79-90, 2018.

[134] F. Mao, Y. Wu, X. Tang et al., "Exosomes derived from human umbilical cord mesenchymal stem cells relieve inflammatory bowel disease in mice," BioMed Research International, vol. 2017, Article ID 5356760, 12 pages, 2017.

[135] J. Yang, X. X. Liu, H. Fan et al., "Extracellular vesicles derived from bone marrow mesenchymal stem cells protect against experimental colitis via attenuating colon Inflammation, Oxidative Stress and Apoptosis," PloS one, vol. 10, no. 10, article e0140551, 2015.

[136] W. Han, B. Xie, Y. Li et al., "Orally deliverable nanotherapeutics for the synergistic treatment of colitis-associated colorectal cancer," Theranostics, vol. 9, no. 24, pp. $7458-$ 7473, 2019.

[137] Z. Davoudi, N. Peroutka-Bigus, B. Bellaire et al., "Intestinal organoids containing poly(lactic-co-glycolic acid) nanoparticles for the treatment of inflammatory bowel diseases," Journal of biomedical materials research Part A, vol. 106, no. 4, pp. 876-886, 2018.

[138] B. Xiao, H. Laroui, S. Ayyadurai et al., "Mannosylated bioreducible nanoparticle-mediated macrophage-specific TNF- $\alpha$ RNA interference for IBD therapy," Biomaterials, vol. 34, no. 30, pp. 7471-7482, 2013.

[139] J. L. Hood, "Post isolation modification of exosomes for nanomedicine applications," Nanomedicine, vol. 11, no. 13, pp. 1745-1756, 2016.

[140] J. L. Hood and S. A. Wickline, "A systematic approach to exosome-based translational nanomedicine," Wiley interdisciplinary reviews Nanomedicine and nanobiotechnology, vol. 4, no. 4, pp. 458-467, 2012.

[141] K. Le Doare, B. Holder, A. Bassett, and P. S. Pannaraj, "Mother's milk: a purposeful contribution to the development of the infant microbiota and immunity," Frontiers in Immunology, vol. 9, p. 361, 2018.

[142] M. Turfkruyer and V. Verhasselt, "Breast milk and its impact on maturation of the neonatal immune system," Current Opinion in Infectious Diseases, vol. 28, no. 3, pp. 199-206, 2015.

[143] W. A. Walker and R. S. Iyengar, "Breast milk, microbiota, and intestinal immune homeostasis," Pediatric Research, vol. 77, no. 1-2, pp. 220-228, 2015.

[144] C. Admyre, S. M. Johansson, K. R. Qazi et al., "Exosomes with immune modulatory features are present in human breast milk," Journal of immunology, vol. 179, no. 3, pp. 19691978, 2007.

[145] T. Hata, K. Murakami, H. Nakatani, Y. Yamamoto, T. Matsuda, and N. Aoki, "Isolation of bovine milk-derived microvesicles carrying mRNAs and microRNAs," Biochemical and Biophysical Research Communications, vol. 396, no. 2, pp. 528-533, 2010.

[146] S. Kahn, Y. Liao, X. Du, W. Xu, J. Li, and B. Lonnerdal, "Exosomal micro RNAs in milk from mothers delivering preterm infants survive in vitro digestion and are taken up by human intestinal cells," Molecular Nutrition \& Food Research, vol. 62, article e1701050, 2018.

[147] Y. Liao, X. Du, J. Li, and B. Lonnerdal, "Human milk exosomes and their microRNAs survive digestion in vitro and are taken up by human intestinal cells," Molecular Nutrition \& Food Research, vol. 61, no. 11, 2017.

[148] A. Hock, H. Miyake, B. Li et al., "Breast milk-derived exosomes promote intestinal epithelial cell growth," Journal of Pediatric Surgery, vol. 52, no. 5, pp. 755-759, 2017.

[149] T. Chen, M. Y. Xie, J. J. Sun et al., "Porcine milk-derived exosomes promote proliferation of intestinal epithelial cells," Scientific Reports, vol. 6, no. 1, article 33862, 2016.

[150] H. N. Gao, H. Y. Guo, H. Zhang, X. L. Xie, P. C. Wen, and F. Z. Ren, "Yak-milk-derived exosomes promote proliferation of intestinal epithelial cells in an hypoxic environment," Journal of Dairy Science, vol. 102, no. 2, pp. 985-996, 2019. 
[151] A. Lucas and T. J. Cole, "Breast milk and neonatal necrotising enterocolitis," Lancet, vol. 336, no. 8730-8731, pp. 15191523, 1990.

[152] S. Zamrik, F. Giachero, M. Heldmann, K. O. Hensel, S. Wirth, and A. C. Jenke, "Impact of an in-house pediatric surgery unit and human milk centered enteral nutrition on necrotizing enterocolitis," BioMed Research International, vol. 2018, Article ID 5042707, 6 pages, 2018.

[153] R. J. Schanler, P. A. Burns, S. A. Abrams, and C. Garza, "Bone mineralization outcomes in human milk-fed preterm infants," Pediatric Research, vol. 31, no. 6, pp. 583-586, 1992.

[154] C. Martin, M. Patel, S. Williams, H. Arora, and B. Sims, "Human breast milk-derived exosomes attenuate cell death in intestinal epithelial cells," Innate Immunity, vol. 24, no. 5, pp. 278-284, 2018.

[155] S. Shandilya, P. Rani, S. K. Onteru, and D. Singh, "Small interfering RNA in milk exosomes is resistant to digestion and crosses the intestinal barrier in vitro," Journal of Agricultural and Food Chemistry, vol. 65, no. 43, pp. 9506-9513, 2017.

[156] J. Kumar and A. M. J. Newton, "Rifaximin-chitosan nanoparticles for inflammatory bowel disease (IBD)," Recent Patents on Inflammation \& Allergy Drug Discovery, vol. 11, no. 1, pp. 41-52, 2017.

[157] P. García-Manrique, G. Gutiérrez, and M. C. Blanco-López, "Fully artificial exosomes: towards new theranostic biomaterials," Trends in Biotechnology, vol. 36, no. 1, pp. 10-14, 2018. 


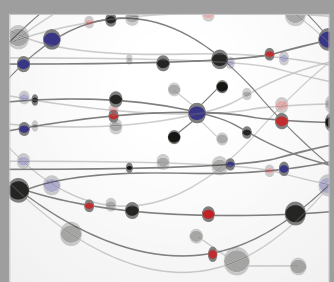

The Scientific World Journal
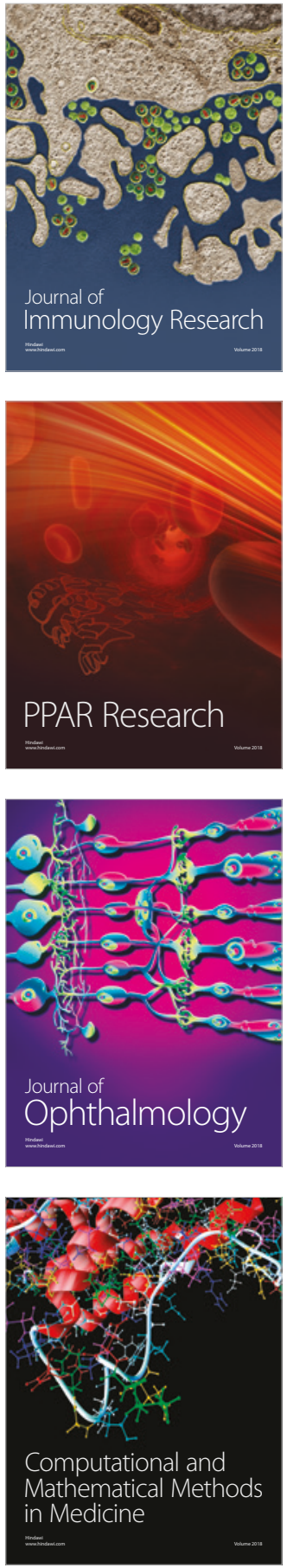

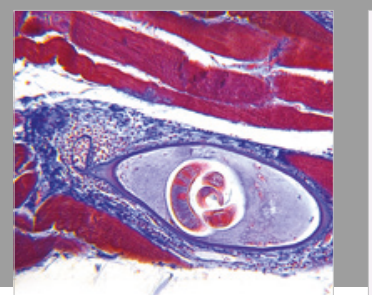

Gastroenterology Research and Practice

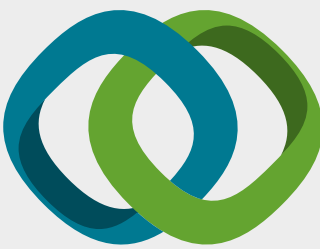

\section{Hindawi}

Submit your manuscripts at

www.hindawi.com
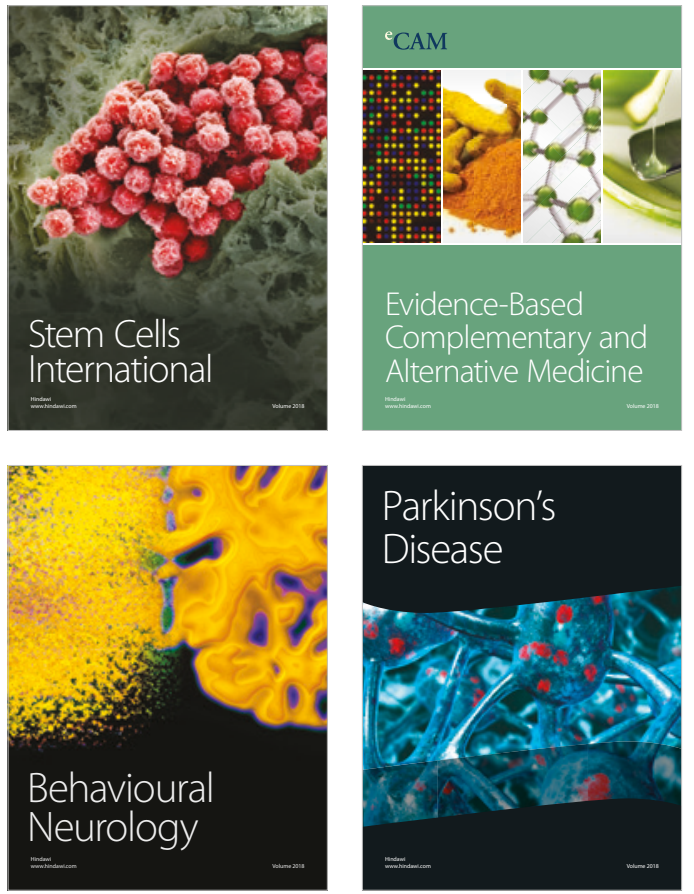

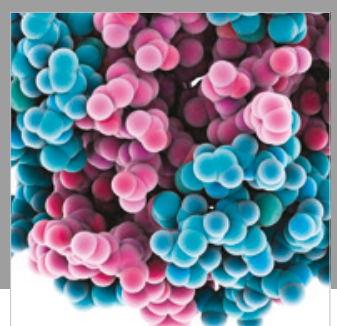

ournal of

Diabetes Research

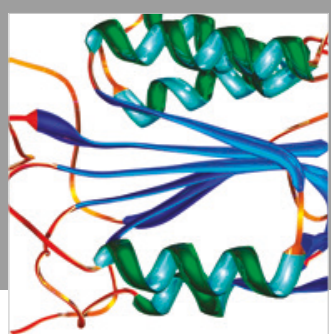

Disease Markers
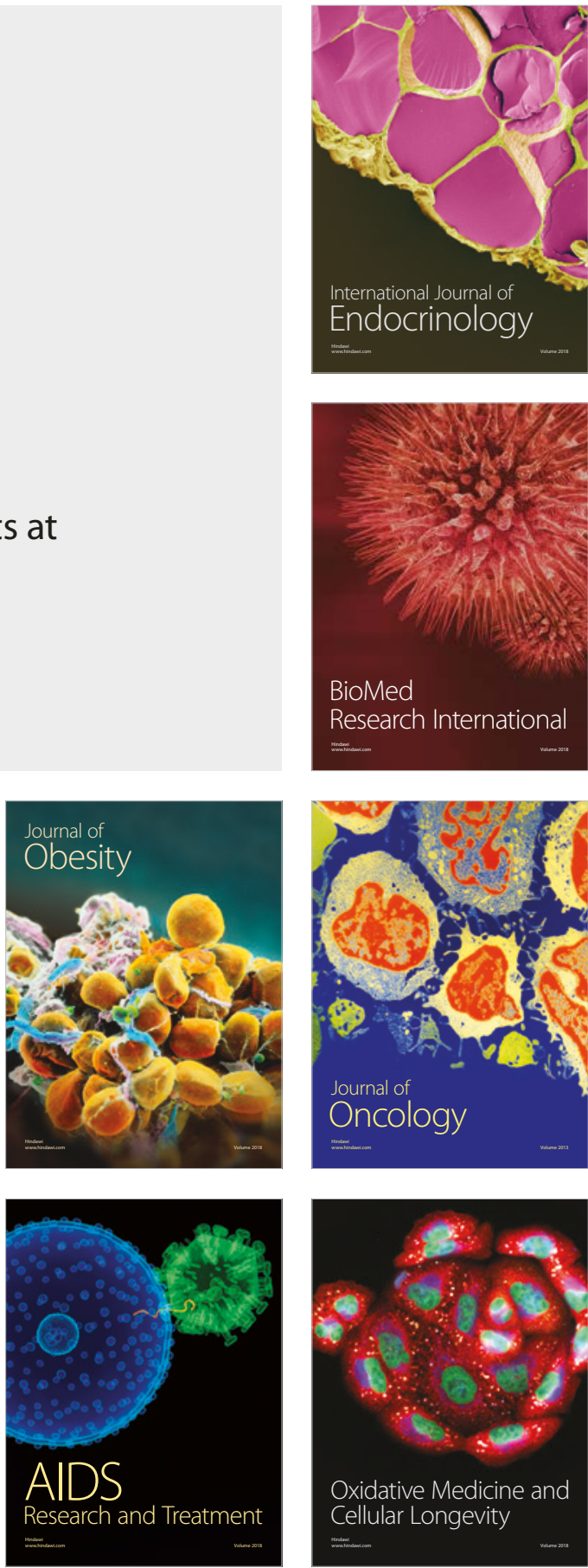